\title{
Double stochastic wave models of multidimensional random fields
}

\author{
V R Krasheninnikov ${ }^{1}$, A U Subbotin ${ }^{1}$ \\ ${ }^{1}$ Department of Applied Mathematics and Informatics, Ulyanovsk State Technical University, \\ Ulyanovsk, Russia \\ e-mail: kvrulstu@mail.ru
}

\begin{abstract}
The paper deals with the development of mathematical models of random fields to describe and simulate images. In the wave model, a random field is the result of the influence of perturbations (waves) that occur at random times in random places and have random shapes. This model allows representing and simulate isotropic and anisotropic images (and their temporal sequences) defined on arbitrary areas of multidimensional space, as well as on any surfaces. The problems of correlation analysis and synthesis can be relatively easily solved. However, this model allows representing only homogeneous fields. In this paper, we consider «double stochastic» wave models, when the first wave random field (control field) sets the parameters of the second (controlled field). As a result, the controlled field becomes nonuniform, since its parameters vary randomly. We also consider options when two fields mutually influence each other. These models allow us to represent and simulate multidimensional inhomogeneous images (and their temporal sequences), as well as systems of such images with mutual correlations.
\end{abstract}

\section{Introduction}

A rigorous mathematical formulation of image processing tasks is required for their effective solution. This formulation primarily includes the model of the image as an object of study. The representation of images by random fields (RF) is generally accepted. To date, there is an extensive literature on image models (or RF models), for example, [1-6]. Much attention is paid to the modeling and processing of medical images for example, [7-11]. However, the vast majority of works consider twodimensional flat RF, less often their sequence. Therefore, it is rather difficult to solve the problems of image imitation, descriptions of inhomogeneous images, correlation analysis and synthesis. A wave model of a random field was proposed in [12-14], which makes it possible to describe homogeneous images and their sequences defined on regions and surfaces of any dimension with small computational costs for simulation. In this model, the RF is the result of the influence of perturbations (waves) that occur at random times in random places and have random shapes. The generated fields are isotropic if waves are spherical. Anisotropic RF can be obtained, for example, with ellipsoidal waves. The main influence on the form of the correlation function of RF is exerted by only one parameter, namely the probability distribution of the wave scale factor, therefore, the problems of correlation analysis and synthesis are relatively easy to solve. 
However, this model describes only homogeneous RF. In $[15,16]$, a double stochastic autoregressive model of RF was proposed for describing inhomogeneous images. In this model, the first wave RF (control field) sets the parameters of the second RF (controlled field), which turns out to be non-uniform, since its parameters randomly vary in space. So the structure of the resulting image is significantly different in different places. This idea is used in the present paper to the wave model, which made it possible to obtain models of a wide class of inhomogeneous images defined on regions and surfaces of any dimensions.

\section{Basic wave model of a random field}

Let us consider the RF wave model [12] that generalizes a number of other models and helps to solve the tasks of analysis and synthesis effectively. This model is simple enough and can serve as a basis for simulating images and their sequences with given covariance function $(\mathrm{CF})$ without increasing the number of model parameters.

In the wave model, the RF is determined by the stochastic equation

$$
x_{\bar{j}}^{t}=\sum_{\left\{k: \tau_{k} \leq t\right\}} f\left[(\bar{j}, t),\left(\bar{u}_{k}, \tau_{k}\right), \bar{\omega}_{k}\right]
$$

where an $(n+1)$-dimensional domain $\{(\bar{j}, t)\}$ may be discrete or continuous, $\left\{\left(\bar{u}_{k}, \tau_{k}\right)\right\}$ is a discrete field of random points (FRP) in an (n+1)-dimensional continuous space, $t$ and $\tau_{k}$ are interpreted as time, $\bar{\omega}_{k}$ is a random vector of function $f$ parameters. This field can be represented as the effect of random disturbances or waves $f\left[(\bar{j}, t),\left(\bar{u}_{k}, \tau_{k}\right), \bar{\omega}_{k}\right]$ appearing in random places $\bar{u}_{k}$ at random time $\tau_{k}$ and changing according to a given law in time and space. Selection of function $f$, the FRP parameters and $\bar{\omega}$ allow us to obtain a vast class of fields.

Let us consider a particular case of a wave model, for which correlation tasks of analysis and synthesis can easily be solved:

$$
f\left[(\bar{j}, t),\left(\bar{u}_{k}, \tau_{k}\right), \bar{\omega}_{k}\right]=g\left(\rho_{k} / R_{k}\right) \exp \left(-\mu /\left|t-\tau_{k}\right|\right) \xi_{k}
$$

where the FRP is a Poisson one with constant density $\lambda, \rho_{k}=\left|\bar{j}-\bar{u}_{k}\right|$ is a distance between $\bar{j}$ and $\bar{u}_{k}$, $\left\{R_{k}\right\}$ is a set of independent non-negative equally distributed random variables density $w(\alpha),\left\{\xi_{k}\right\}$ is a system of independent equally distributed random variables with zero mean. Waves are motionless, independent, have spherical sections in space and exponentially attenuate over time. System $\left\{\xi_{k}\right\}$ determines a wave intensity and $\left\{R_{k}\right\}$ is their spatial scale. If $g(y)=c \exp \left(-2 y^{2}\right)$ then the generated field $X$ is stationary, homogeneous, has zero mean and its CF

$$
V(\rho, t)=\frac{c^{2} \pi^{n / 2} \lambda}{2^{n+1}} \exp (-\mu t) \int_{0}^{\infty} \alpha^{n} \exp \left(-\rho^{2} / \alpha^{2}\right) w(\alpha) \mathrm{d} \alpha
$$

is spatially isotropic with variance

$$
\sigma_{n}^{2}=\frac{c^{2} \pi^{n / 2} \lambda}{2^{n+1} \mu} M\left[R^{n}\right]
$$

Simulation of a discrete field on an $n$-dimensional grid $S=\{\bar{j}\}$ with time quantization $\Delta t$ can be implemented by the following algorithm. At the initial time $t_{0}=0$, the field values in all nodes are equal to zero. At each subsequent moment $t_{m}=m \Delta t$, a Poisson FRP with density $\lambda \Delta t$ is formed over continuous space or grid, which somehow overlaps $S$. At each generated point $\bar{u}_{k}$, random values $\xi_{k}$ and $R_{k}$ are formed. After that the following transformation of all field values on grid $\{\bar{j}\}$ is carried out:

$$
x_{j}^{t_{m}}=x_{j}^{t_{m-1}} \exp (-\mu \Delta t)+c \sum_{k} \exp \left(\rho_{k} / R_{k}\right) \xi_{k},
$$

which forms a sequence of frames that are n-dimensional images defined on $S$. 
Note that the field values are calculated independently of each other. This makes it possible to simulate images on the desired area $S$, for example, on a certain surface.

An example of image simulation with the described wave model is represented in figure 1 . It is necessary to underline that (5) actually implements time-varying images. Therefore, this figure shows only one frame of this process.

Each field value in (5) is a sum of random numbers of the random variables. Thus, generally speaking, the field will not be Gaussian even with Gaussian $\left\{\xi_{k}\right\}$. However, when the model parameter $h=\lambda M\left[R^{n}\right] / \mu$ grows, then the number of summands (5) with similar distributions increase and the field is normalized.

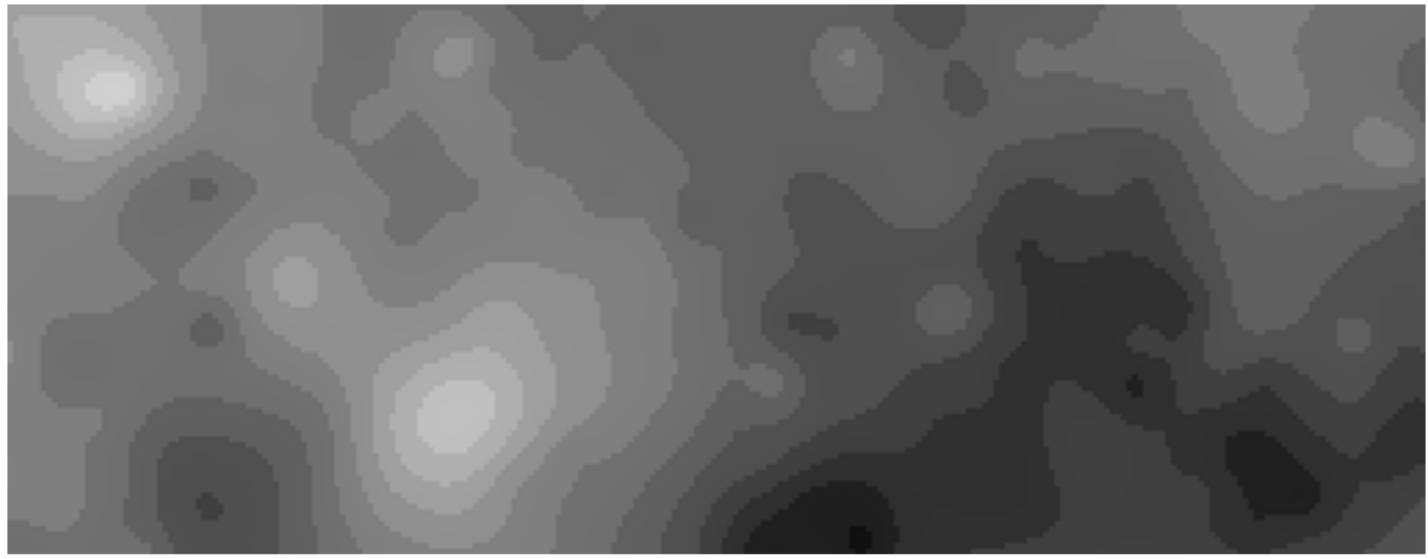

Figure 1. Example of image simulation using a wave model.

Now consider the solution of correlation analysis and synthesis task. It follows (4) that the formed field has an exponential time normalised $\mathrm{CF}(\mathrm{NCF}) e^{-\mu t}$ and space NCF.

$$
r(\rho)=\frac{1}{M\left[R^{n}\right]} \int_{0}^{\infty} \alpha^{n} \exp \left(-\rho^{2} / \alpha^{2}\right) \omega(\alpha) \mathrm{d} \alpha
$$

Thus, solving analysis tasks, when the density $\omega(\alpha)$ is given, the required NCF can be found analytically or by numerical integration. Solving synthesis tasks, when the NCF $r(\rho)$ is given, it is necessary to solve integral equation (6) with respect to unknown $\omega(\alpha)$. As it is not always possible to find an analytical solution of (6), we consider a method of its approximate solution. It follows from (2) that in the case of degenerate distribution $\left(R=\alpha=\right.$ const) we obtain the NCF equal to $\exp \left(-\rho^{2} / \alpha^{2}\right)$. Let now an arbitrary non-increasing NCF $r(\rho)$ be given. Let us approximate it with adequate accuracy by a sum of Gaussoids with positive coefficients $r(\rho) \approx h(\rho)=\sum_{i} q_{i} \exp \left(-\rho^{2} / \alpha_{i}^{2}\right)$, where $\sum_{i} q_{i}=1$, when $r(0)=1$. Then, for discrete distribution $P\left(R=\alpha_{i}\right)=k^{-1} q_{i} / \alpha_{i}^{n}$, where $k=\sum_{i} q_{i} / \alpha_{i}^{n}$, the generated field will have NCF equal to $h(\rho)$. Thus, the generated model allows us approximately to solve a synthesis task by changing only the density of scale $R$.

\section{Double stochastic wave models}

The wave model described above only defines homogeneous images. For the formation of inhomogeneous images, it is necessary that the model parameters vary in the domain of $S$. This can be achieved by breaking $S$ into parts, in each of which the wave model has its own set of parameters. But, taking into account the possible uncertainty of real images, "double stochastic" models are considered in [6, 7], in which one or several autoregressive (control) RF set the parameters of the final (controlled) autoregressive RF. We use this idea for wave models. 
Let the control field $X$ be given by model (2) with some values of its parameters. We use for the controlled field $Y$ a model of the same type but with a modified density $\omega(\alpha)$ depending on $X$. For example,

$$
R_{k}^{Y}=r_{m}+\left(x^{t}\left(\bar{u}_{k}\right)-\min X^{t}\right) R_{k},
$$

where $X^{t}$ is the field $X$ at the time t, $\bar{u}_{k}$ is formed the point of FRP for $Y^{t}, x^{t}\left(\bar{u}_{k}\right)$ is field value at this point, $r_{m}$ is the minimum allowable scale value $R_{k}^{Y}$ for $Y, R_{k}^{Y}$ is random scale value (when it is distributed for the field $X$ ). Transformation (7) leads to the corresponding conversion $w_{X}(\alpha) \rightarrow w_{Y}(\alpha)$ of the $Y$ field scale, which should be used in (6) when calculating the CF of field $Y$.

Note that other doubly stochastic models are possible when other parameters of the resulting image are also possible (c value, FRP density, and so on) are determined by one or several control images.

Figure 2 (b) shows the image $Y$, controlled by the top image $X$ in figure 2 (a). It is noticeable that the smoother areas of the image $Y$ correspond to the brighter areas of the image $X$, that is, its larger values and larger values of the wave stretch factor $R_{k}^{Y}$. The sample value of the interval of absolute correlation in a smoother region of this image is 87 , and at the less smooth region is only 12 .

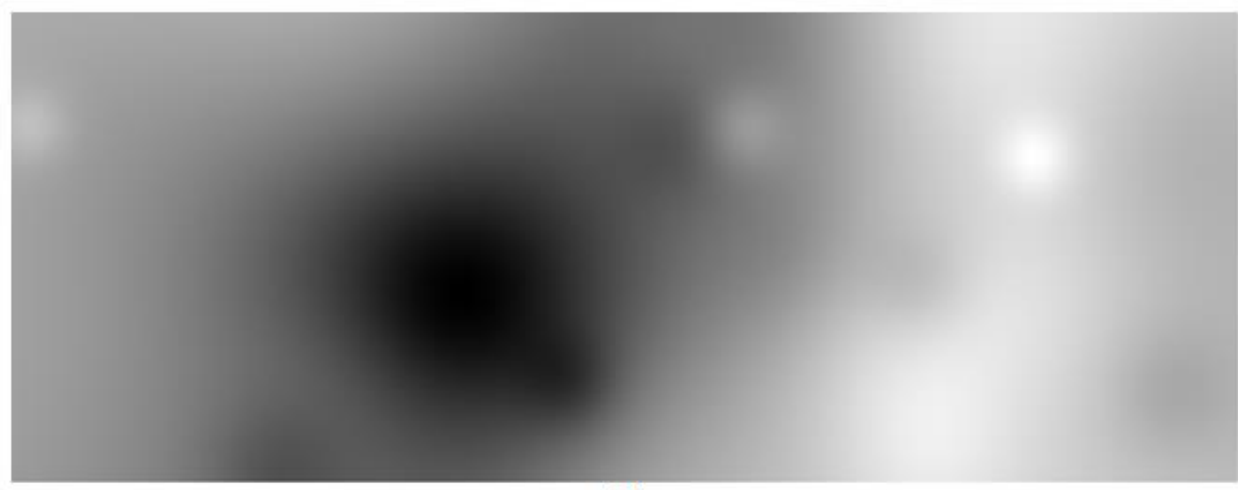

(a)

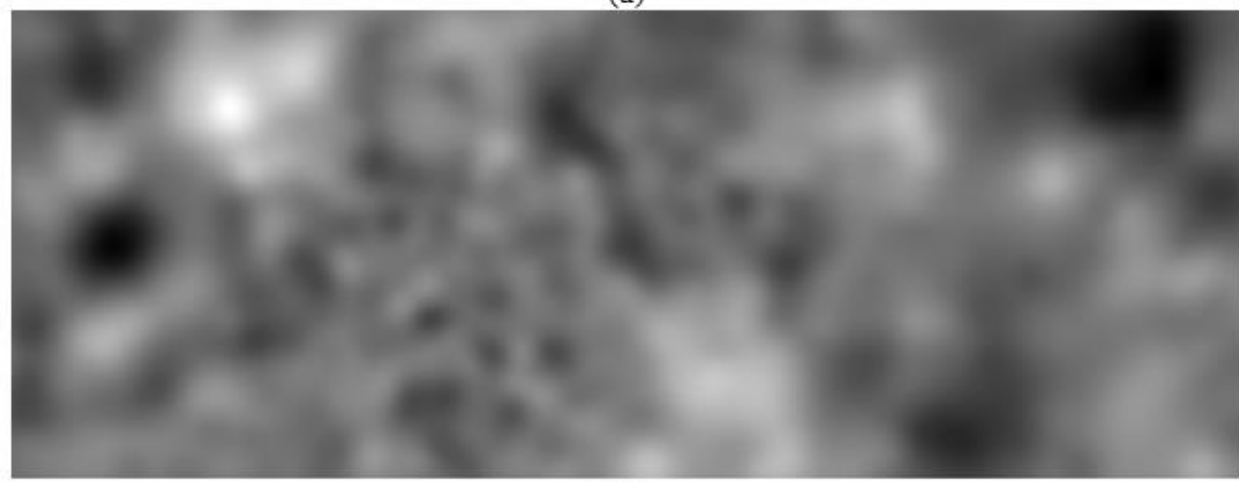

(b)

Figure 2. An example of simulating double stochastic images: (a) is a control image, (b) is a controlled image.

However, the boundaries between more and less smooth areas on $Y$ are blurred, since the waves have a considerable length and the brightness $X$ varies smoothly. In [6], to obtain clearer boundaries on double-stochastic autoregressive images, the quantization of the control image is applied, which provides a stepwise change in the parameters of the controlled one. This method can also be applied to wave models. Figure 3 (a) shows a 3-level quantized image in figure 2 (a). The image controlled by this quantized image is shown in figure 3 (b). Here, the boundaries between areas of different smoothness are clearer than in figure 2(b). The sample values of the correlation interval are approximately the same as in figure 2 (b). 


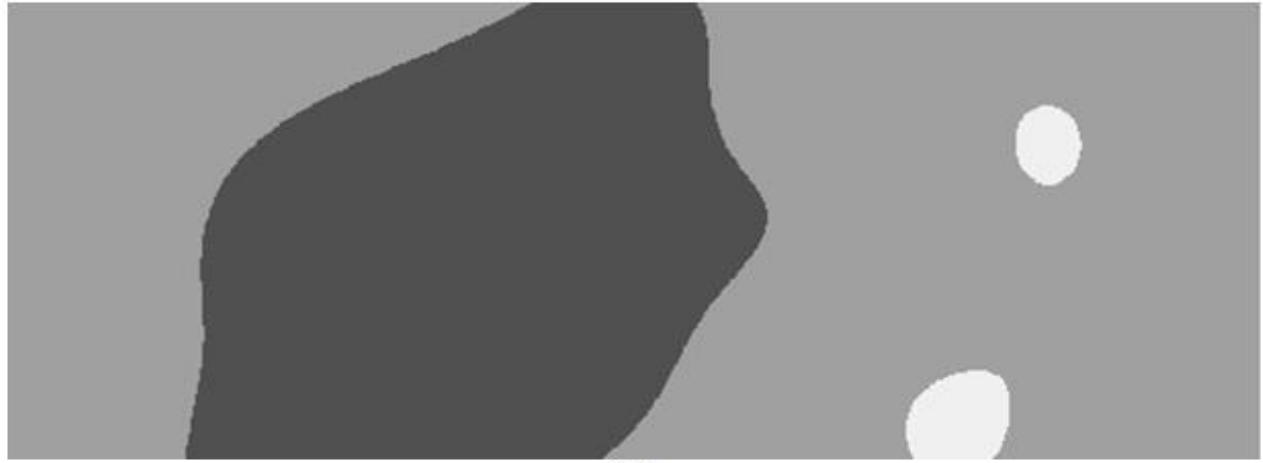

(a)

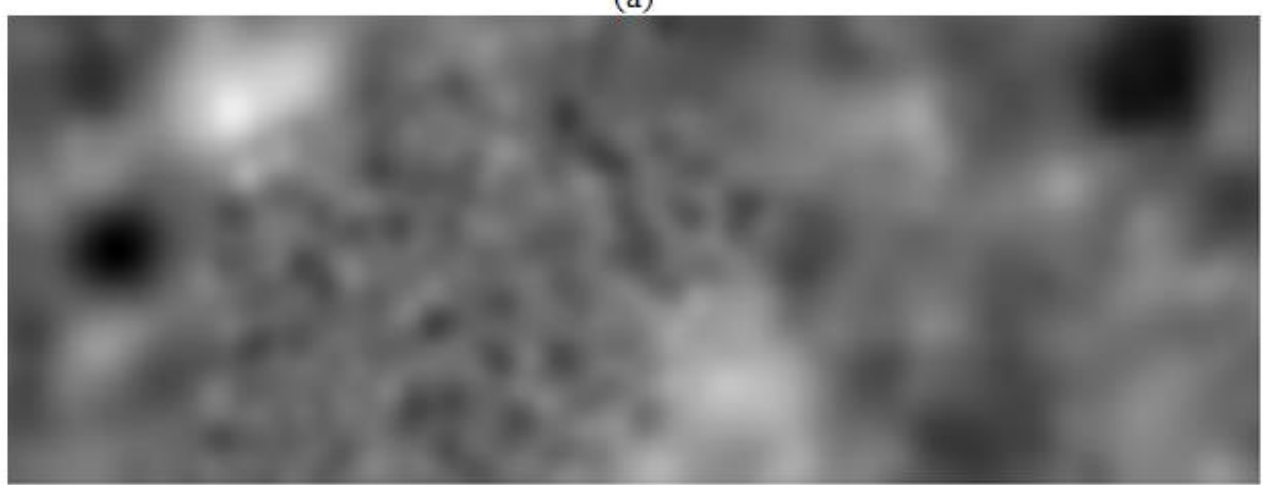

(b)

Figure 3. An example of simulating double-stochastic images with quantization: (a) is a control image, (b) is a controlled image.

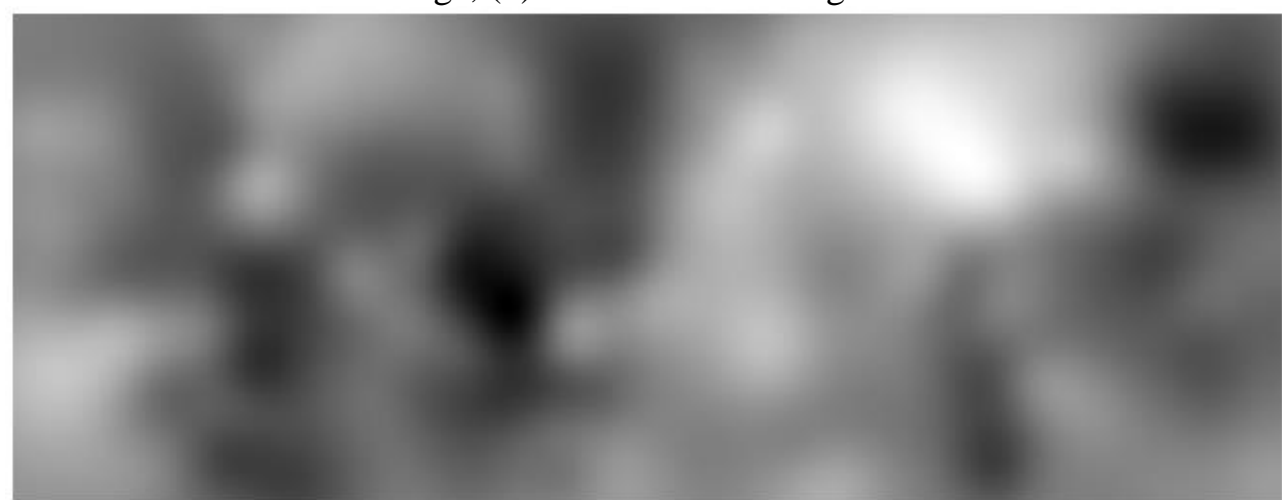

(a)

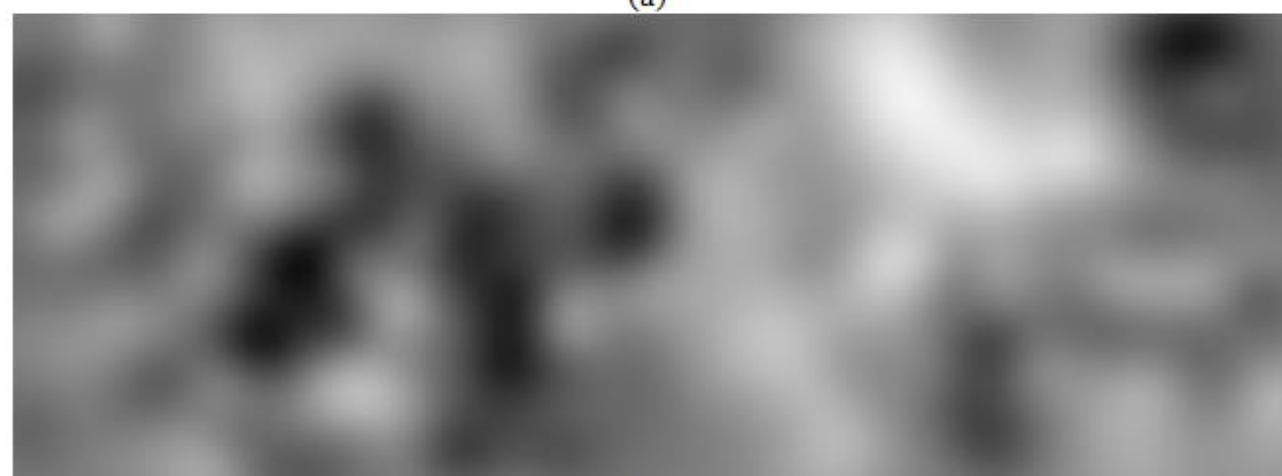

(b)

Figure 4. An example of simulating double stochastic images with mutual influence. 
Two images are unequal in the described model: one controls the parameters of the other. In [8], a model of autoregressive images defined on a cylinder was proposed, jointly controlling the parameters of each other. We apply this approach to the considered wave models. Consider the sequence $X_{1}, X_{2}, X_{3}, \ldots$ of images. Images $X_{1}$ and $Y_{1}$ are formed in the same way as $X$ and $Y$ in the model described above. Image $X_{2}=a X_{1}+b Y_{1}$ controls the formation of the image $Y_{2}$, and so on. Here should be $|a|<1$. This process is similar to autoregression. As a result, in the steady state, the frames of the formed sequence mutually control the parameters of each other. The correlation weakens with an increase in the time interval between frames. Figure 4 shows two consecutive images formed in the manner described. Their correlation is visually noticeable (the sample correlation coefficient is 0.769 ). This is significantly different from simple control (figure 2), where the correlation between the images is almost zero.

\section{Conclusions}

This paper presents double stochastic wave models of random fields (images). In these models, the control wave random field sets the parameters of the controlled random field. As a result, the structure of the controlled image turns out to be significantly different in different places, which makes it possible to describe inhomogeneous images with random inhomogeneities. Options are also considered when two or more fields mutually influence each other. These models allow to represent and simulate multidimensional inhomogeneous images (and their temporal sequences), as well as systems of such images with mutual influences.

\section{References}

[1] Soifer V A, Popov S B, Mysnikov V V and Sergeev V V 2009 Computer image processing. Part I: Basic concepts and theory (VDM Verlag Dr.. Muller) p 300

[2] Vizilter Y V, Pyt'ev Y P, Chulichkov A I and Mestetskiy L M 2015 Morphological image analysis for computer vision applications Computer Vision in Control Systems-1, ISRL 73 (Springer International Publishing Switzerland) 9-58

[3] Shalygin A S and Palagin Y I 1986 Applied methods of statistical modeling (Mechanical engineering, American society of mechanical engineers) p 320

[4] Gonzalez R C and Woods R E 2002 Digital image processing (Prentice Hall, Upper Saddle River) p 976

[5] Myasnikov V V 2018 Description of images using a configuration equivalence relation Computer Optics 42(6) 998-1007 DOI: 10.18287/2412-6179-2018-42-6-998-1007

[6] Labunets V G, Koch E V and Ostkhaymer E 2018 Algebraic models and methods of computer image processing. Part 1. Multiplet models of multichannel images Computer Optics 42(1) 8495 DOI: $10.18287 / 2412-6179-2018-42-1-84-95$

[7] Bourne R 2010 Fundamentals of Digital Imaging in Medicine (London: Springer) p 200

[8] Ammari H (ed) 2012 Mathematical Modeling in Biomedical Imaging II: Optical, Ultrasound, and Opto-Acoustic Tomographies (Springer) p 173

[9] Shirokanev A S, Kirsh D V, Ilyasova N Yu and Kupriyanov A V 2018 Investigation of algorithms for coagulate arrangement in fundus images Computer Optics 42(4) 712-21 DOI: 10.18287/2412-6179-2018-42-4-712-721

[10] Khorin P A, Ilyasova N Yu and Paringer R A 2018 Informative feature selection based on the Zernike polynomial coefficients for various pathologies of the human eye cornea Computer Optics 42(1) 159-166 DOI: 10.18287/2412-6179-2018-42-1-159-166

[11] Krasheninnikov V R and Kopylova A S 2012 Algorithms for automated processing images of blood serum facies Pat Rec Im An 22 583-592

[12] Krasheninnikov V R and Vasil'ev K K 2018 Multidimensional image models and processing Computer Vision in Control Systems-3, ISRL 135 (Springer International Publishing Switzerland) 11-64 
[13] Krasheninnikov V R 2012 Models of random fields on surfaces Proceedings of the Samara Scientific Center of the Russian Academy of Sciences 4 812-816

[14] Krasheninnikov V R and Subbotin A Yu 2018 Doubly stochastic model of a quasi-periodic process as an image on a cylinder Proceedings of the International Scientific and Technical Conference "Advanced Information Technologies" (PIT-2018) (Samara: Samara Scientific Center of the Russian Academy of Sciences) 1017-1021

[15] Vasilyev K K and Dement'iev V E 2017 Presentation and processing of satellite multi-zone images (Ulyanovsk: Ulyanovsk State Technical University) p 251

[16] Vasil'ev K K, Dement'ev V E and Andriyanov N A 2015 Doubly stochastic models of images Pat Rec Im An 25 105-110 